\section{IDDF2021-ABS-0085 ASSOCIATION OF HEALTHY AND UNHEALTHY PLANT-BASED DIETS WITH THE RISK OF COLORECTAL CANCER OVERALL AND BY MOLECULAR SUBTYPES}

${ }^{1}$ Fenglei Wang*, ${ }^{2}$ Tomotaka Ugai, ${ }^{2}$ Koichiro Haruki, ${ }^{1} Y_{i}$ Wan, ${ }^{2}$ Andrew Chan, ${ }^{1}$ Stephanie Smith-Warner, ${ }^{1}$ Xuehong Zhang, ${ }^{1}$ Edward Giovannucci, ${ }^{1}$ Walter Willett, ${ }^{2}$ Shuji Ogino. 'Harvard T.H. Chan School of Public Health, USA; ${ }^{2}$ Brigham and Women's Hospital and Harvard Medical School, USA

\subsection{6/gutjnl-2021-IDDF.131}

Background Several plant foods have been associated with a lower risk of colorectal cancer (CRC). However, not all plant foods are healthy, and little is known about the association between plant-based diets and specific molecular subtypes of CRC. We aimed to examine the association of healthy and unhealthy plant-based diets and the risk of CRC overall and by molecular subtypes.

Methods We followed 76,386 women in the Nurses' Health Study (1984-2014) and 47,387 men in the Health Professionals Follow-up Study (1986-2014) for up to 30 years. Plantbased diets were assessed using two indices derived from the food frequency questionnaires: a healthy plant-based diet index (hPDI) and an unhealthy plant-based diet index (uPDI). Colorectal tumor tissues were collected to measure the status of microsatellite instability (MSI), CpG island methylator phenotype (CIMP), and BRAF and KRAS mutations.

Results During 3,143,205 person-years of follow-up, we documented 3077 incident CRC cases, including 1244 cases with available molecular marker data. A higher hPDI was associated with lower CRC incidence (hazard ratio [HR] comparing extreme quartiles $=0.86,95 \% \mathrm{CI}$ : 0.77, 0.96; P-trend $=0.04$ ), whereas a higher uPDI was associated with higher CRC incidence (HR comparing extreme quartiles $=1.16$, 95\% CI: 1.04, 1.29; P-trend=0.005). The association of hPDI significantly differed by KRAS status (P-heterogeneity $=0.003$ ) but not by other tumor markers. The hPDI was associated with lower incidence of KRAS-wildtype CRC (HR comparing extreme quartiles $=0.74$, 95\% CI: 0.57, 0.96; P-trend=0.004) but not KRAS-mutant CRC (P-trend=0.22).

Conclusions A plant-based diet rich in whole grains, fruits, and vegetables was associated with a lower incidence of CRC, especially KRAS-wildtype CRC, whereas a plant-based diet emphasizing unhealthy plant foods such as refined grains might be associated with higher CRC incidence. These data emphasize the importance of differentiating healthy and unhealthy plant foods when promoting plant-based diets for the primary prevention of CRC. The differential association of hPDI by KRAS status suggests the interplay of diet and specific tumor pathways.

\section{IDDF2021-ABS-0086 DIVERSIFIED ASPECTS OF DONOR SCREENING FOR WASHED MICROBIOTA TRANSPLANTATION AT CHINA MICROBIOTA TRANSPLANTATION SYSTEM}

Gaochen Lu*, Pan Li, Faming Zhang. Medical Center for Digestive Diseases, The Second Affiliated Hospital of Nanjing Medical University, Nanjing, China

10.1136/gutjnl-2021-IDDF.132

Background Effective screening of donors can greatly improve the safety and efficacy of washed microbiota transplantation (WMT), which is of great concern to the public. fmtBank (www.fmtbank.org), a non-profit public project providing national rescue WMT, needs to meet the continuous demand for qualified fecal donors based on the automatic microfiltration machine. Therefore, we have established a step-by-step donor screening program and management process.

Methods The qualification of 944 candidates to serve as potential WMT donors was assessed from 2015 to 2021 in Nanjing Medical University. Individuals interested in fecal donation should complete a three-stage donor-screening process, including questionnaire interview (IDDF2021-ABS0086 Table 1), face-to-face interview and laboratory testing.

Results Figure 1 and 2 shows the whole process of screening (IDDF2021-ABS-0086 Figure 1. Crowd demonstrated, IDDF2021-ABS-0086 Figure 2. Screening process). In total 898 valid questionnaires, the age was $20.77 \pm 1.45$. Only $8.7 \%$ meet the inclusion and exclusion criteria. 82 potential donors who have passed the primary screening need to undergo a face-to-face interview with a trained physician, which selected 54 students to finish laboratory tests including blood, urine and fecal screening. Finally, 32 qualified donors were selected. Because the median time a donor actively donated stool in our center was 11.5 months, we defined stool donation for more than 6 months as a long-term donor. 10 students

Abstract IDDF2021-ABS-0086 Table 1 Inclusion and exclusion criteria for primary screening

Inclusion criteria

(1) Willing to finish questionnaire screening

(2) Healthy children and adults under 60 years old, preferably 6-24 years old (3) Normal body mass index $\left(18-24 \mathrm{~km} / \mathrm{m}^{2}\right)$ and regular bowel habits

Exclusion criteria

(1) History of medication

Took antimicrobial (antibiotics, antifungals, antivirals), probiotics, laxative within 3 months Took immunosuppressive medications (eg., Calcineurin inhibitors, biological agents, exogenous glucocorticoids) within 6 months

Took proton pump inhibitors more than 1 month

(2) History of diseases

History of infection disease (eg., HIV, HBV or HCV, syphilis, human T-lymphotropic virus and II, malaria, trypanosomiasis, tuberculosis), gastrointestinal disease (eg., change in bowel habits and polyposis), systemic immune disease

Atopic disease (eg., asthma, eczema, eosinophilic disorders of the gastrointestinal tract) Metabolic syndrome, obesity or moderate to severe undernutrition/malnutrition; Chronic pain syndromes (eg., chronic fatigue syndrome, fibromyalgia) neurologicl neurodevelopmental disorders

History of malignant illness

Psychologic diseases

(3) Family history

Family history of gastrointestinal cancer involving 3 or more first and second degree of relatives

Family history of autoimmune and metabolic diseases involving 3 or more first and second degree of relatives

Household members with active gastrointestinal infection

(4)Sex behaviors

High-risk sexual behavior (anonymous sexual contacts; sexual contacts with prostitutes, drug addicts, individuals with HIV, viral hepatitis, syphilis; work as prostitutes; history of sexually transmittable disease )

HIV: Human immunodeficiency virus: HBV: Hepatitis B virus; HCV: Hepatitis C virus 
eventually became long-term donors. 32 donors provided 2009 times of fecal donations. As shown in Figure 3 (IDDF2021-ABS-0086 Figure 3. Correlation of fecal weight and amount of enriched washed microbiota), the fecal weight was not well correlated with the amount of enriched washed microbiota (95\% CI, 0.62-0.67, P $<0.0001$ ), r was 0.64 , (IDDF2021-ABS-0086 Figure 4. Detailed exclusion criteria and proportions).

Conclusions In our center, we selected 32 donors from 944 students, with a qualified rate of $3.4 \%$. This result is similar to the qualified rate of donor screening reported in stool

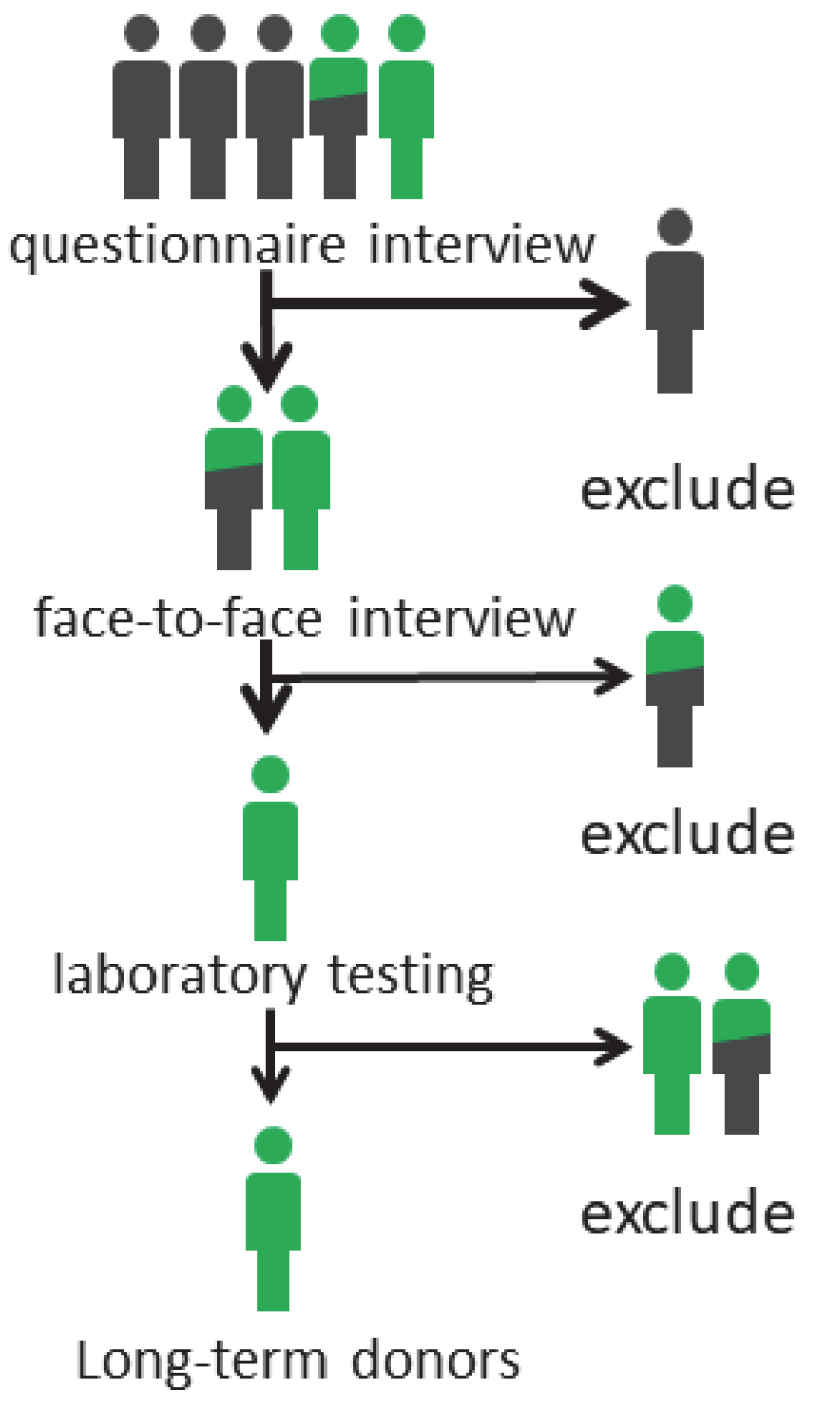

Abstract IDDF2021-ABS-0086 Figure 1

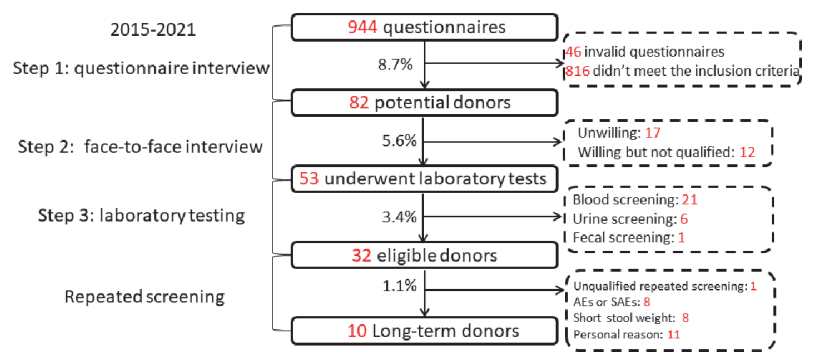

Abstract IDDF2021-ABS-0086 Figure 2

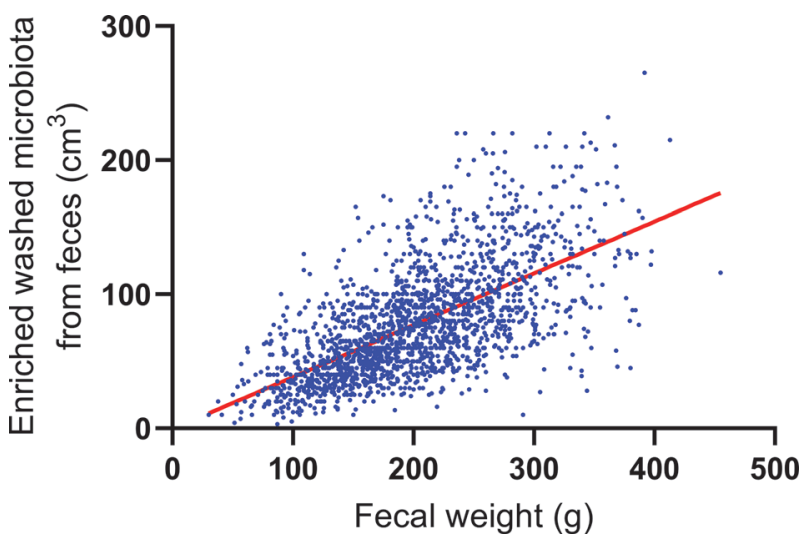

Abstract IDDF2021-ABS-0086 Figure 3

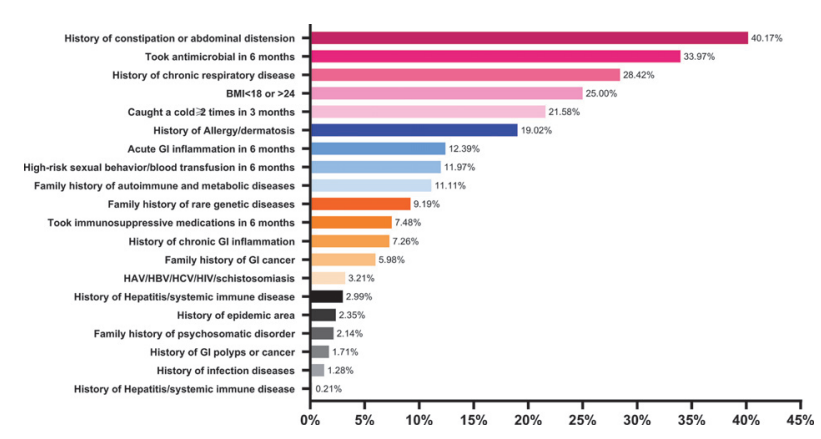

Abstract IDDF2021-ABS-0086 Figure 4

bank of the United States, Canada and South Korea. And we found that long-term donors have better microbiota output and compliance. The study found that it is feasible to screen qualified donors from a large sample by step-by-step screening. Through this programmed donor screening management process, convenient and repeatable donor screening can be realized.

\section{IDDF2021-ABS-0087 ENTEROCOCCUS AND BIFIDOBACTERIUM MAY BE RISK FACTORS FOR FECAL MICROBIOTA TRANSPLANTATION-RELATED ADVERSE EVENTS}

Weihong Wang*, Pan LI, Xia Wu, Faming Zhang. The Second Affiliated Hospital of Nanjing Medical University, China

\subsection{6/gutjnl-2021-IDDF.133}

Background Fecal microbiota transplantation (FMT) for inflammatory bowel disease (IBD) is an effective means of treatment, adverse events (AE) may influence the use of FMT and make patients worry about its safety. At present, there are still few studies on the safety of FMT in the world. Most of the previous studies on the safety of FMT focus on the gut microbiota from donors but ignore its from patients. This study is the first to analyze the risk factors of the occurrence and clinical efficacy of FMT-related AE from the perspective of patients.

Methods 102 IBD patients who had received FMT were included in the study. Clinical efficacy was followed up for 3 months and $\mathrm{AE}$ occurred during the course of disease was 